\title{
Calidad de vida en adolescentes: Evaluación de necesidades basada en factores personales
}

\section{Quality of life in adolescents: Needs assessment based on personal factors.}

\author{
Jesús Miguel Muñoz-Cantero *, Luisa Losada-Puente ** \\ *Universidade da Coruña, **Universidade da Coruña.
}

\begin{abstract}
Resumen
Este trabajo explora la influencia de diversos factores personales (sexo, edad y presencia de NEAE) sobre la calidad de vida de la adolescencia. Mediante metodología cuantitativa, se aplica Cuestionario de Evaluación de la Calidad de Vida de Alumnos adolescentes (CCVA, Gómez-Vela y Verdugo, 2009) a 2220 estudiantes (12-19 años). Los resultados muestran mayor satisfacción con la vida en alumnado varón y más joven experimenta, y peor en alumnado con NEAE. Las tres variables estudiadas, de forma independiente, contribuyen a explicar las diferencias, pero no conjuntamente. Se propone continuar el estudio analizando la influencia de variables ambientales (familia, escuela).

Palabras clave: calidad de vida, adolescencia, evaluación de necesidades
\end{abstract}

\section{Abstract}

This work explores the influence of various personal factors (sex, gender and presence of SEN) on the quality of life of adolescence. Using quantitative methodology, the Questionnaire for Assessment of the Quality of Life of adolescent Students (QAQL, Gómez-Vela \& Verdugo, $2009)$ is applied to 2220 students (12-19 years). The results show more satisfaction with life in male and youngest students, and worst in students with SEN. The three studied variables, independently, contribute to explain the differences, but not together. It is proposed to continue the study by analyzing the influence of environmental variables (family, school).

Keywords: quality of life, adolescence, needs assessment.

\section{Introducción}

La calidad de vida ocupa un lugar central en la investigación en Ciencias Sociales, desde donde se define como concepto multidimensional que recoge las valoraciones objetivas y las percepciones subjetivas del individuo acerca de las diversas áreas que componen a su vida. Se trata, según Urzúa y Caqueo-Urízar (2012), de un "estado de bienestar general que comprende descriptores objetivos y evaluaciones subjetivas de bienestar físico, material, social y emocional, junto con el desarrollo de actividades, todas estas mediadas por los valores personales" (p.64). Bajo esta definición subyace la idea de la calidad de vida como un concepto integrado por múltiples factores e indicadores que evalúan el grado en que las personas tienen experiencias vitales que valoran y el modo en que éstas contribuyen a una vida plena para ellas (Schalock et al., 2002).

\section{Calidad de vida en la adolescencia: un marco teórico para su evaluación.}

En el terreno educativo, este constructo se sitúa como eje para la evaluación de los resultados personales y para el diseño de intervenciones que pretenden la mejora académica, personal y social del alumnado y también la de las escuelas (Muntaner, 2013). En este sentido, evaluar la calidad de vida de la adolescencia permite atender a las áreas que son de mayor importancia para la juventud y detectar aquellas en las que es necesario incidir para garantizar su bienestar emocional, físico y material, su integración y su presencia en la comunidad, sus relaciones interpersonales, su desarrollo personal y su autodeterminación.

Estas dimensiones son reflejadas en el Modelo de Calidad de Vida en la adolescencia (Gómez-Vela y Verdugo, 2004, 2006) que desarrolla un marco conceptual para la medición y aplicación de la calidad de vida en la adolescencia con y sin Necesidades Específicas de Apoyo Educativo (en adelante, NEAE), bajo el enfoque de la multidimensionalidad y de la influencia de las características individual y las variables interpersonales en su configuración.

Evaluación de la calidad de vida en la adolescencia: Producción científica actual.

Un creciente número de publicaciones se centran en evaluar la calidad de vida en la adolescencia, haciendo hincapié en los factores condicionantes sobre su promoción, tanto a nivel individual (sexo, edad, presencia o no de NEAE) como ambiental (escuela, familia, entorno comunitario). Mayoritariamente, dichas investigaciones centran su atención en analizar elementos vinculados a la salud y las psicopatologías en la infancia y la adolescencia (Adelman, Taylor y Nelson, 1989;Huebner, 1991; Levesque, 2014; Sylverster, Donnell, Gray, Higgins y Stalker, 2015) y son menos las que estudian la satisfacción y la felicidad percibida por la juventud en las áreas psicológica, social y educativa (Alfaro, Casas y López, 2015; Casas, Castellá et al., 
2012; Casas, Sarriera, et al., 2012; Higuita y Cardona, 2015;Leversen, Danielsem, Birkeland y Samlad, 2012).

También se observa insuficiente la producción científica que evalúa la calidad de vida en la adolescencia con NEAE o centrada en la influencia de los apoyos educativos sobre su mejora, si bien se hace evidente que el profesorado de Educación Secundaria Obligatoria (ESO) se enfrenta al reto de ofrecer una respuesta adecuada a las necesidades de un número cada vez mayor de alumnado que requiere atención educativa específica, al tiempo que debe ajustar la enseñanza a un currículum común, homogéneo y poco flexible (Domínguez y Vázquez, 2015; Miranda, Burguera y Arias, 2015), lo que puede repercutir sobre la calidad de la enseñanza y sobre la calidad de los apoyos y ayudas que se presten a cada alumno para mejorar su bienestar y satisfacción.

Por todo ello, el objetivo de este trabajo es explorar y describir la percepción de la adolescencia acerca de su calidad de vida, atendiendo a los principales factores personales que pueden incidir sobre su configuración.

\section{Método}

Se trata de un estudio de corte cuantitativo, de tipo descriptivo-exploratorio, en el que se delinean variables personales relacionadas con el nivel de calidad de vida percibido por la adolescencia. La calidad de vida actúa como variable dependiente, evaluada a través del Cuestionario de Evaluación de la Calidad de Vida de Alumnos adolescentes (CCVA, Gómez-Vela y Verdugo, 2009) y definida en base a siete dimensiones. Por su parte, las variables independientes consideradas son el sexo, la edad y la presencia de NEAE.

\section{Instrumento}

El Cuestionario de Evaluación de la Calidad de Vida de Alumnos adolescentes (CCVA, Gómez-Vela y Verdugo, 2009) se compone de 66 ítems, con formato de respuesta tipo Likert de 4 puntos. Está diseñado para su aplicación a adolescentes de 12-18 años con y sin NEAE. Evalúa siete dimensiones de la calidad de vida (bienestar emocional, integración en la comunidad, relaciones interpersonales, desarrollo personal, bienestar físico, autodeterminación y bienestar material) fundamentadas en el Modelo de Calidad de Vida (Gómez-Vela y Verdugo, 2004, 2006).

Para este estudio, el análisis de la validez del instrumento, mediante un AFE, permite extraer siete dimensiones que explican el $53.24 \%$ de la varianza (superior a la obtenida en la validación inicial: $51 \%$ ) pero revela ciertas diferencias en la estructura factorial, quedando formada por las dimensiones: bienestar emocional y físico, bienestar material, inclusión, relaciones interpersonales, desarrollo personal, derechos y autodeterminación. Dicha estructura y el cuestionario en general presentanbuenos índices de fiabilidad $(\alpha=.917$; rango $\alpha=.915-.796)$.

\section{Muestra}

La muestra se compone de 2220 estudiantes de 12-19 años $(\mathrm{M}=14.29$; D.T.=1.81) con y sin NEAE, de los cuales 1230 son hombres $(55.41 \%)$ y 990 mujeres (44.59\%) y existen 746 alumnos con NEAE (33.6\%) y
1474 sin ellas (66.4\%). Es una muestra intencional y por conveniencia, teniendo como criterios los siguientes:

- Que el alumnado estuviese escolarizado en Centros de Educación Ordinaria o Específica de la Comunidad Autónoma de Galicia

- Que tuviesen entre 12-19 años, y cursasen los estudios correspondientes a la Educación Secundaria Obligatoria, Bachillerato o Formación Profesional.

- Que tuviesen habilidades de lectoescritura y un nivel medio de comprensión lectora o, en su ausencia, que constasen con los apoyos suficientes para cumplimentar el cuestionario.

El procedimiento de recogida de datos fue el siguiente:

- Se contactó con centros educativos de la Comunidad Autónoma de Galicia vía e-mail para solicitar su participación en el estudio.

- Los centros interesados en participar recibieron un documento de confidencialidad, a firmar por el responsable del centro (director) y familiares del alumnado.

- La aplicación del instrumento se realizó a nivel de aula (grupos de 20-25 alumnos) o en grupos reducidos (8-10 alumnos). El alumnado con necesidad de apoyo de carácter intensivo y extenso, recibieron ayuda del personal investigador $\mathrm{y}$ profesionales del centro.

\section{Procedimiento de análisis}

Dado el cumplimiento de los supuestos de normalidad y homocedasticidad, se emplearon como técnicas estadísticas la Prueba T-Student, el Análisis de Varianza Simple (ANOVA) y Análisis Multivariante de la Varianza (MANOVA). Mediante la prueba T-Student, se comparó la calidad de vida global, estableciendo comparaciones en función del sexo y la presencia o no de NEAE. A través de la prueba ANOVA se estudiaron las diferencias en calidad de vida en función de tres grupos de edad (12-13 años, 14-15 años, y 16 ó más años) en base al cálculo de los valores percentiles, que permitieron crear grupos de edad más homogéneos. Finalmente, se aplicó la prueba MANOVA incluyendo todas las dimensiones de la calidad de vida, para comprobar las diferencias entre hombres y mujeres, y presencia o no de NEAE, controlando la variable edad.

\section{Resultados}

En primer lugar, se presenta las puntuaciones medias y la desviación típica de cada dimensión de la escala y del total, diferenciando por sexo, edad y presencia o no de NEAE (Tabla 1).

Tabla 1.

Estadísticos descriptivos: Media y Desviación típica

\begin{tabular}{ccccccccc}
\hline & \multicolumn{2}{c}{ Sexo } & \multicolumn{3}{c}{ Edad } & \multicolumn{3}{c}{ NEAE } \\
\hline & $\mathrm{H}$ & $\mathrm{M}$ & $12-13$ & $14-15$ & 16 ó + & $\mathrm{Si}$ & $\mathrm{No}$ \\
\hline \multirow{2}{*}{ BEF } & .38 & -.48 & .82 & .07 & -1.3 & -.49 & .25 \\
& $(3.1)$ & $(3.4)$ & $(2.9)$ & $(3.3)$ & $(3.3)$ & $(3.4)$ & $(3.2)$ \\
BMA & .03 & -.04 & .28 & -.01 & -39 & -.33 & .17 \\
& $(2.4)$ & $(2.3)$ & $(2.24)$ & $(2.3)$ & $(2.5)$ & $(2.5)$ & $(2.3)$
\end{tabular}




\begin{tabular}{lccccccc} 
INC & .09 & -.12 & .46 & .09 & -.77 & -.30 & .15 \\
& $(2.7)$ & $(2.5)$ & $(2.6)$ & $(2.72)$ & $(2.42)$ & $(2.9)$ & $(2.6)$ \\
RRI & .04 & -.05 & .49 & -.15 & -54 & -.30 & .15 \\
& $(2.8)$ & $(2.7)$ & $(2.6)$ & $(2.9)$ & $(2.8)$ & $(2.9)$ & $(2.6)$ \\
DEP & -.07 & -.04 & .5 & -.02 & -.69 & -.19 & .09 \\
& $(2.1)$ & $(2.1)$ & $(2.01)$ & $(2.2)$ & $(2.1)$ & $(2.12)$ & $(2.1)$ \\
DER & -.10 & -.001 & -.17 & .09 & .14 & -.46 & .23 \\
& $(2.1)$ & $(1.9)$ & $(2.1)$ & $(1.9)$ & $(2.1)$ & $(2.3)$ & $(1.9)$ \\
\multirow{2}{*}{ AUT } & -.55 & .68 & -1.67 & 1.12 & 1.12 & -5.7 & 2.9 \\
& $(1.7)$ & $(1.6)$ & $(1.7)$ & $(1.6)$ & $(1.7)$ & $(1.9)$ & $(1.5)$ \\
\multirow{2}{*}{ Total } & .31 & -.39 & 2.2 & .19 & -3.3 & -2.6 & 1.3 \\
& $(10.7)$ & $(10.2)$ & $(10.1)$ & $(10.6)$ & $(10.1)$ & $(11.3)$ & $(9.8)$ \\
\hline
\end{tabular}

Nota: siglas BEF-Bienestar emocional y físico, BMA-Bienestar material, INC-Inclusión, RRI-Relaciones Interpersonales, DEP-Desarrollo Personal, DER-Derechos, AUT-Autodeterminación.

A través del estadístico T-Student se comparan las diferencias entre hombres y mujeres en la valoración de su calidad de vida, teniendo en cuenta que a nivel descriptivo parecen existir diferencias a favor de los hombres. Para un nivel de confianza del $95 \%$, dichas diferencias no resultan estadísticamente significativas $\left(t_{157.16}=1.56 ; p=.118\right)$. En cuanto a sus dimensiones, existen diferencias estadísticamente significativas en bienestar emocional y físico $\left(t_{2218}=6.19 ; p \leq .000\right)$ a favor de los varones, y en derechos $\left(t_{2218}=--2.5 ; p<.013\right)$ a favor de las mujeres.

También se observan diferencias estadísticamente significativas entre alumnado con y $\sin \operatorname{NEAE}(F=19.92$; $\left.p \leq .000 ; \quad t_{2218}=-8.492 ; \quad \mathrm{p}<.000\right)$ siendo superior en alumnado sin NEAE, manteniéndose estas diferencias en todas las dimensiones contrastadas.

La prueba ANOVA estudia la presencia de diferencias entre alumnado de tres grupos de edad en su percepción de la calidad de vida y sus dimensiones. Los resultados muestran diferencias estadísticamente significativas en la percepción de la calidad de vida en función de la edad $(F=52.32 ; p \leq .000)$ y en todas sus dimensiones $(p<.05)$.

La tabla 2 presenta los resultados de la prueba Post-Hoc, que evidencia la presencia de diferencias entre prácticamente todos los grupos de edad conformados, a excepción de las dimensiones derechos y autodeterminación donde no se aprecian diferencias estadísticamente significativas entre los grupos de 14-15 años y de 16 o más, por lo que se puede decir que la pertenencia al intervalo de edad de 14 o más años evidencia una percepción de calidad de vida similar, aunque diferente respecto a la del grupo de 12-13 años.

En todos los contrastes efectuados, la tendencia es a valorar de forma más negativa la calidad de vida cuanto mayor edad tiene el adolescente.

Estos resultados señalan que el alumnado de 12-13 años tiende a valorar de forma más positiva su calidad de vida y, con ella, también su bienestar emocional y físico, inclusión, relaciones interpersonales, bienestar material, desarrollo personal y derechos. El alumnado de mayor edad (16 o más años) presenta una calidad de vida significativamente inferior $(F=52.315 ; p \leq .000)$ al resto de grupos. Al contrario sucede en el caso de la autodeterminación donde el alumnado de 12-13 años puntúa significativamente por debajo, no detectándose diferencias entre alumnado de 14-15 años y 16-17 años.

Tabla 2.

Comparaciones múltiples: Pruebas Post-Hoc Scheffé.

\begin{tabular}{|c|c|c|c|c|c|c|c|}
\hline \multirow[t]{2}{*}{ VD } & \multirow{2}{*}{$\begin{array}{l}\text { (I) } \\
\text { Edad }\end{array}$} & \multirow{2}{*}{$\begin{array}{l}\text { (J) } \\
\text { Edad }\end{array}$} & \multirow{2}{*}{$\begin{array}{c}\text { Dif. } \\
\text { medias } \\
(\mathrm{I}-\mathrm{J})\end{array}$} & \multirow[t]{2}{*}{ E.T. } & \multirow[t]{2}{*}{ Sig. } & \multicolumn{2}{|c|}{ IC 95\% } \\
\hline & & & & & & L.inf. & L.Sup \\
\hline \multirow{6}{*}{ CDV } & \multirow{2}{*}{$12-13$} & $14-15$ & $2.00^{*}$ & .53 & .00 & .77 & 3.24 \\
\hline & & 16_19 & $5.59^{*}$ & .55 & .00 & 4.30 & 6.88 \\
\hline & \multirow{2}{*}{$14-15$} & $12-13$ & $-2.01^{*}$ & .53 & .00 & -3.24 & -.77 \\
\hline & & 16_19 & $3.59^{*}$ & .58 & .00 & 2.24 & 4.94 \\
\hline & \multirow{2}{*}{ 16_19 } & $12-13$ & $-5.59^{*}$ & .55 & .00 & -6.88 & -4.31 \\
\hline & & $14-15$ & $-3.59^{*}$ & .58 & .00 & -4.94 & -2.24 \\
\hline \multirow{6}{*}{ BEF } & \multirow{2}{*}{$12-13$} & $14-15$ & $.75^{*}$ & .16 & .00 & .38 & 1.12 \\
\hline & & 16_19 & $2.09^{*}$ & .17 & .00 & 1.70 & 2.48 \\
\hline & \multirow{2}{*}{$14-15$} & $12-13$ & $-.75^{*}$ & .16 & .00 & -1.12 & -.38 \\
\hline & & 16_19 & $1.34^{*}$ & .17 & .00 & .93 & 1.75 \\
\hline & \multirow{2}{*}{ 16_19 } & $12-13$ & $-2.09^{*}$ & .17 & .00 & -2.48 & -1.70 \\
\hline & & $14-15$ & $-1.34^{*}$ & .17 & .00 & -1.75 & -.93 \\
\hline & \multirow{2}{*}{$12-13$} & $14-15$ & $.36^{*}$ & .13 & .02 & .060 & .67 \\
\hline & & 16_19 & $1.22^{*}$ & .13 & .000 & .91 & 1.53 \\
\hline & \multirow{2}{*}{$14-15$} & $12-13$ & $-.36^{*}$ & .13 & .020 & -.67 & -.06 \\
\hline & & 16_19 & $.86^{*}$ & .14 & .000 & .53 & 1.19 \\
\hline & \multirow{2}{*}{ 16_19 } & $12-13$ & $-1.22^{*}$ & .13 & .000 & -1.53 & -.91 \\
\hline & & $14-15$ & $-.86^{*}$ & .14 & .000 & -1.19 & -.53 \\
\hline \multirow{6}{*}{ RRI } & & $14-15$ & $.64^{*}$ & .14 & .000 & .32 & .96 \\
\hline & $12-13$ & 16_19 & $1.04^{*}$ & .14 & .000 & .70 & 1.37 \\
\hline & & $12-13$ & $-.64^{*}$ & .14 & .00 & -.96 & -.32 \\
\hline & $14-15$ & 16_19 & $.39^{*}$ & .15 & .03 & .05 & .75 \\
\hline & & $12-13$ & $-1.04^{*}$ & .14 & .00 & -1.37 & -.70 \\
\hline & 16_19 & 14- & $-.39^{*}$ & .1 & .03 & -.75 & -.05 \\
\hline & & 14 & $.29^{*}$ & .1 & .0 & .02 & .57 \\
\hline & $12-13$ & 16 & $.67^{*}$ & .12 & .00 & .39 & .96 \\
\hline & & $12-13$ & $-.29^{*}$ & .12 & .04 & -.57 & -.02 \\
\hline$\sqrt{11}$ & $14-1 J$ & 16_19 & $.38^{*}$ & .13 & .01 & .07 & .68 \\
\hline & & $12-13$ & $-.67^{*}$ & .1 & .00 & -.96 & -.38 \\
\hline & _19 & 14 & $-.38^{*}$ & .13 & .01 & -.68 & -.07 \\
\hline & & $14-15$ & $.52^{*}$ & .1 & .00 & .28 & .77 \\
\hline & 1 & 16_19 & $1.19^{*}$ & .11 & .00 & .93 & 1.44 \\
\hline & & $12-13$ & $-.52^{*}$ & .10 & .00 & -.77 & -.28 \\
\hline D & & 16_19 & $.66^{*}$ & .11 & .00 & .40 & .93 \\
\hline & & $12-13$ & $-1.18^{*}$ & .11 & .00 & -1.4 & -.93 \\
\hline & & $14-15$ & $-.66^{*}$ & .11 & .00 & -.93 & -.40 \\
\hline & & $14-15$ & $-.26^{*}$ & .10 & .04 & -.50 & -.02 \\
\hline & $12-15$ & 16_19 & $-.31^{*}$ & .11 & .02 & -.56 & -.05 \\
\hline & & $12-13$ & $.26^{*}$ & .10 & .04 & .02 & .50 \\
\hline D & & 16_19 & -.05 & .11 & .92 & -.31 & .22 \\
\hline & & $12-13$ & $.31^{*}$ & .11 & .02 & .05 & .56 \\
\hline & 10_19 & $14-15$ & .05 & .11 & .92 & -.22 & .31 \\
\hline & & $14-15$ & $-.22^{*}$ & .08 & .03 & -.42 & -.03 \\
\hline & $12-13$ & 16_19 & $-.22^{*}$ & .09 & .04 & -.42 & -.02 \\
\hline & & $12-13$ & $.22^{*}$ & .08 & .03 & .02 & .42 \\
\hline AU1 & $14-15$ & 16_19 & .001 & .09 & 1.0 & -.21 & .21 \\
\hline & & $12-13$ & $.22^{*}$ & .09 & .04 & .02 & .42 \\
\hline & 16_19 & $14-15$ & -.001 & .09 & 1.0 & -.21 & .21 \\
\hline
\end{tabular}

*. La diferencia de medias es significativa al nivel 0.05 .

Finalmente, se analiza la Varianza Factorial con dos factores: sexo y presencia o no de NEAE. De la combinación de estos dos factores suponemos la existencia de cuatro poblaciones normales, independientes y homocedásticas.

Primero, se utiliza la variable calidad de vida como variable dependiente, siendo controlado el efecto de la edad (covariable); posteriormente, se aplica el mismo análisis al estudio de cada una de sus dimensiones. En la 
tabla 3 se observan, en primer lugar, los efectos individuales de los dos factores incluidos en el modelo, junto con el de la covariable edad. Los $p$-valores asociados a cada uno de ellos indican que los varones y las mujeres obtienen resultados diferentes en calidad de vida $(p \leq .000)$, al igual que el alumnado con $\mathrm{y}$ sin necesidades específicas de apoyo educativo $(p \leq .000)$ yel alumnado de los tres grupos de edad $(p \leq .000)$.

Al estudiar la interacción entre ambas variables, se comprueba que el estadístico $F$ asociado a este efecto $(F=.003)$ tiene un nivel crítico $p=.953$, que indica que el efecto de la interacción no es significativo. Finalmente, se observa que el coeficiente de correlación entre los tres efectos incluidos en el modelo (sexo, presencia o no de NEAE y la interacción entre ambos), controlando el efecto de la variable edad son capaces de explicar solamente el $7 \%$ de la calidad de vida.

Tabla 3.

Pruebas de los efectos inter-sujetos. Variable dependiente: Calidad de vida

\begin{tabular}{|c|c|c|c|c|c|}
\hline Origen & $\begin{array}{l}\text { Suma de } \\
\text { cuadrados } \\
\text { tipo III }\end{array}$ & gl & $\begin{array}{c}\text { Media } \\
\text { cuadrática }\end{array}$ & $\mathrm{F}$ & Sig \\
\hline $\begin{array}{l}\text { Modelo } \\
\text { corregido }\end{array}$ & $18231.06^{\mathrm{a}}$ & 4 & 4557.766 & 42.79 & .00 \\
\hline Intersección & 5486.55 & 1 & 5486.554 & 51.51 & .00 \\
\hline Edad & 9373.39 & 1 & 9373.391 & 88.00 & .00 \\
\hline Sexo & 816.57 & 1 & 816.574 & 7.67 & .01 \\
\hline NEAE & 6358.93 & 1 & 6358.929 & 59.70 & .00 \\
\hline Sexo*NEAE & .370 & 1 & .370 & .003 & .9 \\
\hline Error & 235929.25 & 2215 & 106.514 & & \\
\hline Total & 254160.31 & 2220 & & & \\
\hline $\begin{array}{l}\text { Total } \\
\text { corregida }\end{array}$ & 254160.31 & 2219 & & & \\
\hline
\end{tabular}

Por tanto, las variables estudiadas de forma independiente contribuyen a explicar las diferencias en la valoración de la calidad de vida por parte del alumnado, pero no lo hacen de forma conjunta, ni se observa efecto de la variable edad sobre la interacción entre el sexo y la presencia de NEAE.

\section{Discusión}

Evaluar la calidad de vida de la adolescencia exige atender a las diferencias individuales que caracterizan a cada individuo con el fin de ajustar las actuaciones a sus necesidades y demandas específicas. En este sentido, se hace imprescindible disponer de herramientas fiables que permitan obtener una visión global del constructo, así como analizar las áreas que afectan al bienestar del alumnado. El CCVA resulta un instrumento útil a tal fin, con el que poder evaluar y reflexionar sobre las necesidades y demandas de cada estudiante. A través de este instrumento ha sido posible analizar la posible influencia de variables de tipo personal sobre la percepción de la calidad de vida de los jóvenes.

Los resultados de este estudio muestran que los varones adolescentes experimentan una mejor calidad de vida en general, que concuerda con los resultados de estudios previos (Días, Bastos, Marzo y García, 2015; Raboteg-Saric, Brajsa-Zganec y Sakic, 2008), especialmente en bienestar emocional y físico e inclusión (Gilman et al., 2008; Urzúa y Mercado, 2008).

Además, el alumnado más joven valor de forma más positiva su bienestar con la vida en todas sus áreas, siendo coherente con estudios previos donde encuentran una correlación negativa entre la edad y la percepción de la calidad de vida (Gómez-Vela, Verdugo y González-Gil, 2007; Urzúa y Mercado, 2008; Viñas, González, García, Malo y Casas, 2015).

Finalmente, se observa una percepción inferior de calidad de vida en alumnado que presenta NEAE, independientemente del sexo y de la edad. Al respecto de estas conclusiones, algunos estudios concluyen la existencia de una relación débil entre calidad de vida y CI, asegurando que un CI elevado no garantiza una mayor felicidad, ni tampoco la educación especial es un factor de riesgo para la disminución del grado de satisfacción global con la vida (Suldo, Riley y Shaffer, 2006) mientras que otros revelan la influencia de la variable funcionamiento intelectual sobre algunas áreas de la calidad de vida, como por ejemplo, el nivel de autodeterminación (Gómez-Vela, Verdugo, González-Gil, Badía y Wehmeyer, 2012; Chou, 2013; Chou, Wehmeyer, Palmer y Lee, 2016) enfatizando en la importancia del tipo y la calidad de los apoyos ofrecidos desde los centros educativos (Freire y Vieira, 2014).

En base a estos resultados, se plantean dos posibles conclusiones: por un lado, la existencia de una relación entre presencia de NEAE y nivel de calidad de vida; y por el otro lado, que junto con las variables personales, sobre todo el nivel o grado de NEAE, el nivel de calidad de vida pueda estar mediado por otras variables de tipo ambiental, tales como las familiares o las escolares.

\section{Referencias}

Adelman, H.S., Taylor, L. y Nelson, P. (1989). Minors' dissatisfaction with their life circumstances. Child Psychiatry and Human Development, 20(2), 135-147. doi:http://dx.doi.org/10.1007/2.0711660

Alfaro, J., Casas, F. y López, V. (2015). Bienestar en la infancia y adolescencia. Psicoperspectivas: Individuo y Sociedad, 14(1), 1-5.

Casas, F., Castellá, J., Abs, D., Coenders, G., Alfaro, J, Saforcada, E. y Tonon, G. (2012).Subjective indicators of personal well-being among adolescents. Performance and results for different scales in latin-language speaking countries: A contribution to the international debate. Child Indicators Research, 5(1), 1-28. doi: http://dx.doi.org/10.1007/s12187-011-9119-1

Casas, F., Sarriera, J., Alfaro, J., González, M., Malo, S., Bertran, I., Figuer, C., ...Valdenegro, B. (2012). Testing the personal wellbeing index on 12-16 year-old adolescents in 3 different countries with 2 new items. Social Indicators Research, 105(3), 461-482. doi.:http://dx.doi.org/10.1007/s11205-011-9781-1

Chou, Y.-C. (2013). Autism and self-determination: measurement and contrast with other disability groups (Tesis Doctoral). De la base de datos ProQuest PsicINFO (Acceso No. 3559045) 
Chou, Y.-C., Wehmeyer, M.L., Palmer, S.B. y Lee, J. (2016). Comparisons of self-determination among students with autism, intellectual disability, and learning disabilities: A multivariable analysis. Focus on Autism and Other Developmental Disabilities, 14, $1-9$. http://dx.doi.org/10.1177/1088357615625059

Dias, P.C., Bastos, A.S., Marzo, J.C. y García, J.A. (2015). Bienestar, calidad de vida y regulación afectiva en adolescentes portugueses. Atención Primaria, 19(5), 432-439. doi: 10.1016/j.aprim.2015.09.005

Domínguez, J. y Vázquez, E. (2015). Atención a la diversidad: Análisis de la formación permanente del profesorado en Galicia. Revista Nacional $e$ Internacional de Educación Inclusiva, 8(2), 139-152.

Freire, V. y Vieira, S.M.C. (2014). Quality of life in adolescents with disabilities. Revista CEFAC, 16(6), 1953-1961.

doi: http://dx.doi.org/10.1590/1982-0216201416213

Gilman, R., Huebner, E.S., Tian, L., Park, N., O’Byrne, J., Schiff, M.,.. Langknecht, H. (2008). Cross-national adolescent multidimensional life satisfaction reports: Analyses of mean scores and response style differences. Journal of Youth and Adolescence,37(2), 142-154. doi: http://dx.doi.org/10.1007/s10964-007-9172-8

Gómez-Vela, M. y Verdugo, M.A. (2004). Cuestionario de evaluación de la calidad de vida de alumnos de educación secundaria obligatoria: Descripción, validación inicial y resultados obtenidos tras su aplicación en una muestra de adolescentes con discapacidad y sin ella. Siglo Cero: Revista Española Sobre Discapacidad Intelectual, 35(4), 212-517.

Gómez-Vela, M. y Verdugo, M.A. (2006). La calidad de vida en la adolescencia: evaluación de jóvenes con discapacidad y sin ella. En M.A. Verdugo (Ed.), Cómo mejorar la calidad de vida de las personas con discapacidad. Instrumentos y estrategias de evaluación (pp. 77-102). Salamanca: Amarú.

Gómez-Vela, M. y Verdugo, M.A. (2009). Cuestionario de evaluación de la calidad de vida en alumnos adolescentes (CCVA).Madrid: CEPE.

Gómez-Vela, M., Verdugo, M.A., González-Gil, F., Badía, M. y Wehmeyer, M.L. (2012). Assessment of self-determination of Spanish students with intellectual and other educational needs.Journal of Applied Research in Intellectual Disabilities, 23(5), 505-506.

Gómez-Vela, M., Verdugo, M.A. y González-Gil, F. (2007). Calidad de vida y autoconocepto en adolescentes con necesidades educativas especiales y sin ellas. Infancia y Aprendizaje, 30(4), 523-536. doi: http://dx.doi.org/10.1174/021037007782334300

Higuita, L.F. y Cardona, J.A. (2015). Concepto de calidad de vida en la adolescencia: Una revisión crítica de la literatura. Revista CES Psicología, 8(1), 155-168.

Huebner, E.S. (1991b). Initial development of the sudent's life satisfaction scale. School Psychology International, 12(3), 231-240. doi: http://dx.doi.org/10.1177/0143034391123010

Leversen, I., Danielsem, A.G., Birkeland, M.S. y Samdal, O. (2012). Basic psychological need satisfaction in leisure activities and adolescents' life satisfaction. Journal of Youth and Adolescence, 41(12), 1588-1599. doi: http://dx.doi.org/10.1007/s10964-012-9776-5

Levesque, R.J.R. (2014b). Self-determination. En Autor (Ed.), Encyclopedia of adolescence ( $2^{\mathrm{a}}$ ed., pp. 2542-2544).Nueva York, EE.UU.: Springer-Verlag.

Miranda, M., Burguera, J.L. y Arias, J.M. (junio, 2015). La atención a la diversidad en los centros de secundaria: Diseño y propiedades de un cuestionario de opinión dirigido a orientadores. En AIDIPE (Ed.), Investigar con y para la sociedad (Vol. 1, pp-103-11). Cádiz: Bubook.

Muntaner, J.J. (2013). Calidad de vida en la escuela inclusiva. Revista Iberoamericana de Educación, 63, 35-49.

Raboteg-Saric, Z., Brajsa-Zganec, A. y Sakic, M. (2008). Life satisfaction in adolescents: The efecto of perceived family economy status and quality of family and peer relationships. Journal for General, Social Issues, 3, 547-564.

Schalock, R.L., Brown, I., Brown, R.I., Cummins, R.A., Felce, D., Matikka, L, Keith, K.D. y Parmenter, T. (2002).Conceptualization, measurement, and application of quality of life for persons with intellectual disabilities: report of an International Panel of Experts. Journal of Mental Retardation, 40(6), 457-470.

doi: http://dx.doi.org/10.1352/0047-6765(2002)040\%3c04 57:CMAAOQ\%3E2.0.CO;2

Suldo, S.M., Riley, K.N, y Shaffer, E.J. (2006). Academic correlates of children and adolescents' life satisfaction. School Psychology International, 27(5), 567-582. doi: 10.1177/0143034306073411

Sylvester, J., Donnell, N., Gray, S., Higgins, K. y Stalker, K. (2014).A survey of disabled children and young people's views about their quality of life. Disability \& Society, 29(5), 763-777. doi: http://dx.doi.org/10.1080/09687599.2013.848782

Urzúa, A. y Caqueo-Urizar, A. (2012). Calidad de vida: una revisión teórica del concepto. Terapia Psicológica, 30(1), 61-71. doi: http://dx.doi.org/10.4067/S0718-48082012000100006

Urzúa, A. y Mercado, G. (2008). La evaluación de la calidad de vida de los y las adolescentes a través del Kiddo-Kindl. Terapia Psicológica, 26(1), 133-141.

Viñas, F., González, M.., García, Y., Malo, S. y Casas, F. (2015). Coping strategies and styles and their relationship to personal wellbeing in a sample of adolescents. Anales de Psicologia, 31(1), 226-233. doi: http://dx.doi.org/10.6018/analesps.31.1.163681 